\title{
Disruption of Dentate Gyrus Blocks Effect of Visual Input on Spatial Firing of CA1 Neurons
}

\author{
Jong Won Lee, ${ }^{1}$ Woon Ryoung Kim, ${ }^{2}$ Woong Sun, ${ }^{2}$ and Min Whan Jung ${ }^{1}$ \\ ${ }^{1}$ Neuroscience Laboratory, Institute for Medical Sciences, Ajou University School of Medicine, Suwon 443-721, Korea, and ${ }^{2}$ Department of Anatomy, \\ College of Medicine, Korea University, Seoul 136-705, Korea
}

The role of dentate gyrus in hippocampal mnemonic processing is uncertain. One proposed role of dentate gyrus is binding internally generated spatial representation with sensory information on external landmarks. To test this hypothesis, we compared effects of visual input on spatial firing of CA1 neurons in Bax knock-out mice in which dentate gyrus neural circuitry is selectively disrupted. Whereas spatial selectivity of CA1 neuronal firing was significantly higher under normal illumination than complete darkness in wild-type mice, it was similarly low in both illumination conditions in Bax knock-out mice. Also, whereas the spatial location of neuronal firing was more stably maintained in the light than in the dark condition in wild-type mice, it was similarly unstable in both illumination conditions in Bax knock-out mice. These results show that visual input allows selective and stable spatial firing of CA1 neurons in normal animals, but this effect is lost if dentate gyrus neural circuitry is disrupted. Our results provide empirical support for the proposed role of dentate gyrus in aligning internally generated spatial representation to external landmarks in building a unified representation of external space.

\section{Introduction}

Dentate gyrus (DG) receives massive unidirectional projections from the entorhinal cortex (EC) and sends its output projections to CA3 area via mossy fibers. Despite a large body of behavioral, physiological, and theoretical studies, specific roles played by the DG in mnemonic operations of the hippocampus are still unclear (Kesner, 2007; Treves et al., 2008). One proposed function of the DG is binding together spatial and nonspatial information in encoding episodic memories. Characteristics of grid cells found in the medial EC (Hafting et al., 2005; Sargolini et al., 2006) suggest representation of internally generated (i.e., dead reckoning-based) spatial maps in this brain structure that can drive place-specific firing of hippocampal neurons (Leutgeb et al., 2005; O'Keefe and Burgess, 2005; Fuhs and Touretzky, 2006; McNaughton et al., 2006; Witter and Moser, 2006; Gorchetchnikov and Grossberg, 2007; Molter and Yamaguchi, 2008). By contrast, neurons in the lateral EC show little spatially selective firing (Hargreaves et al., 2005), suggesting the segregation of spatial and nonspatial information processing in the medial and lateral EC, respectively (Redish, 1999; Knierim et al., 2006; Eichenbaum et al., 2007; Kerr et al., 2007). Because medial and

\footnotetext{
Received May 25, 2012; revised July 30, 2012; accepted Aug. 1, 2012.

Author contributions: J.W.L. and M.W.J. designed research; J.W.L. performed research; W.R.K. and W.S. contributed unpublished reagents/analytic tools; J.W.L. and M.W.J. analyzed data; J.W.L., W.S., and M.W.J. wrote the paper.

This work was supported by a National Research Foundation grant (2011-0015618), the Original Technology Research Program for Brain Science (2011-0019209 and 2011-0019212), and a grant from the Brain Research Center of the 21st Century Frontier Research Program funded by the Ministry of Education, Science and Technology, Korea.

Correspondence should be addressed to Min Whan Jung, Neuroscience Laboratory, Institute for Medical Sciences, Ajou University School of Medicine, Suwon 443-721, Korea. E-mail: min@ajou.ac.kr.

DOI:10.1523/JNEUROSCI.2608-12.2012

Copyright $@ 2012$ the authors $\quad 0270-6474 / 12 / 3212999-05 \$ 15.00 / 0$
}

lateral EC inputs converge in the DG and CA3, but not in CA1, it has been proposed that internally generated spatial maps are associated with the information on the external landmarks in the DG-CA3 network, forming a distinct spatial context for each environment (Redish and Touretzky, 1997; Hafting et al., 2005; O'Keefe and Burgess, 2005; Knierim et al., 2006; Witter and Moser, 2006; Gorchetchnikov and Grossberg, 2007; Leutgeb and Leutgeb, 2007). Currently, however, empirical evidence for or against this hypothesis is scant.

We set out the current study to test the proposed role of DG in aligning internally generated spatial representation to external landmarks. Humans and animals can keep track of spatial position based on idiothetic as well as allothetic cues (O'Keefe and Nadel, 1978; Gallistel, 1990; Redish, 1999). Spatial navigation solely based on idiothetic cues (dead reckoning) is prone to cumulative error that can be corrected in reference to allothetic cues when they are available (landmark-based navigation) (Gallistel, 1990). Thus, in normal animals, a difference is expected in the stability of hippocampal spatial firing according to the availability of allothetic cues; spatial firing is expected to undergo gradual drift when allothetic cues are unavailable, but to be stable when they are available. In DG-lesioned animals, however, spatial firing is expected to undergo gradual drift regardless of the presence of allothetic cues, if the DG indeed plays a critical role in aligning internally generated spatial representation to external landmarks. To test these predictions, we examined stability of spatial firing of CA1 neurons recorded from wild-type (WT) and Bax knock-out (Bax-KO) mice, in which DG neural circuitry is selectively disrupted (Sun et al., 2004; Kim et al., 2009; Lee et al., 2009), across light and dark conditions. The results were consistent with both predictions, providing empirical evidence for the role of the DG in aligning internally generated spatial maps to external landmarks. 


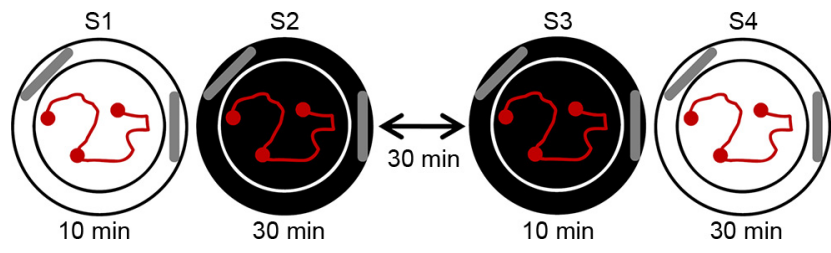

Figure 1. Recording procedure. Single units were recorded in a circular chamber (made of a transparent wall that was surrounded by a black circular curtain containing two visual cues) in the following sequence: light on for $10 \mathrm{~min}$ (session 1; S1), light off for $30 \mathrm{~min}$ (session 2; S2), intersession interval with light off (30 min), light off for $10 \mathrm{~min}$ (session 3; S3), and light on for $30 \mathrm{~min}$ (session 4; \$4).

\section{Materials and Methods}

Animals. Bax-KO mice were maintained on a C57BL/6 background. Homozygous Bax-deficient (Bax-KO) and WT littermate mice were generated from matings between heterozygous males and females. Sibling animals were collected individually and genotyped by PCR (Knudson et al., 1995). All animals were food-deprived to $80 \%$ of their free-feeding body weights and allowed access to water ad libitum. They were maintained on a $12 \mathrm{~h}$ light-dark cycle and all experiments were conducted during the light phase of the cycle. The experimental protocol was approved by the Institutional Animal Care and Use Committee of the Ajou University School of Medicine.

Unit recording. Four tetrodes were chronically implanted in the left or right dorsal hippocampus $(1.7 \mathrm{~mm}$ posterior and $1.5 \mathrm{~mm}$ lateral to bregma) of 10 mice (six WT littermates and four Bax-KO mice, 6-8 months old) under deep anesthesia with sodium pentobarbital $(50 \mathrm{mg} /$ $\mathrm{kg}$, i.p.). Unit signals were recorded from the dorsal CA1 region while the animals were chasing and consuming $20 \mathrm{mg}$ sucrose pellets that were dropped from a dispenser mounted on the ceiling (one pellet every $60 \mathrm{~s}$ ). The recording chamber was a transparent cylinder (diameter, $30 \mathrm{~cm}$; height, $30 \mathrm{~cm}$ ) that was placed on a table (height, $80 \mathrm{~cm}$ ) and surrounded by a black circular curtain (diameter, $100 \mathrm{~cm}$; height, $280 \mathrm{~cm}$ ) containing two white cue cards $(20 \times 40 \mathrm{~cm})$ at 3 and 10 o'clock. White noise was provided through a speaker placed under the recording chamber throughout the entire recording sessions. Same unit signals were recorded under normal illumination (the recording chamber and cue cards were illuminated by two LED lamps mounted on the ceiling; 460 lux) and complete darkness (all lights were off; 0 lux as measured by a light meter; TM 204, Tenmars Electronics) alternatively in the following order: session 1 (10 min, light condition), session 2 (30 min, dark condition), intersession interval (30 min, dark condition), session 3 (10 min, dark condition), and session 4 (30 min, light condition) (Fig. 1). Unit signals were also recorded for $10 \mathrm{~min}$ while the animals sat quietly on a pedestal outside the circular arena before and after the main recording sessions to ensure the stability of recorded unit signals. The animals rested in a cage during the intersession interval, and fresh paper was placed on the floor before sessions 1 and 3 . Unit signals were amplified $10,000 \times$, filtered between 600 and $6000 \mathrm{~Hz}$, digitized at $32 \mathrm{kHz}$, and stored on a personal computer using a Cheetah data acquisition system (Bozemann). The animal's head position was also monitored by tracking an infrared LED mounted on the headstage at $60 \mathrm{~Hz}$. When recordings were completed, small marking lesions were made and recording locations were verified histologically as previously described (Song et al., 2005).

Analysis. Single units were isolated by manually clustering various spike waveform parameters (MClust, A. D. Redish, University of Minnesota, Minneapolis, MN). Recorded units were classified into complex spike cells (mean firing rate $<5 \mathrm{~Hz}$, spike width $>250 \mu \mathrm{s}$, and occasional bursts) and theta cells (the rest) (Lee et al., 2009), and only complex spike cells were included in the analysis. Spatial firing rate maps were constructed by dividing the $67 \times 50 \mathrm{~cm}$ rectangle containing the circular arena into $80 \times 60$ pixels and calculating firing rate for each pixel using adaptive binning as described previously (Jung et al., 1994). A place field was defined as a group of 10 or more adjoining pixels with the average firing rate of each pixel exceeding 3 SDs above the mean firing rate (Song et al., 2005). Spatial selectivity of unit firing was assessed by calculating
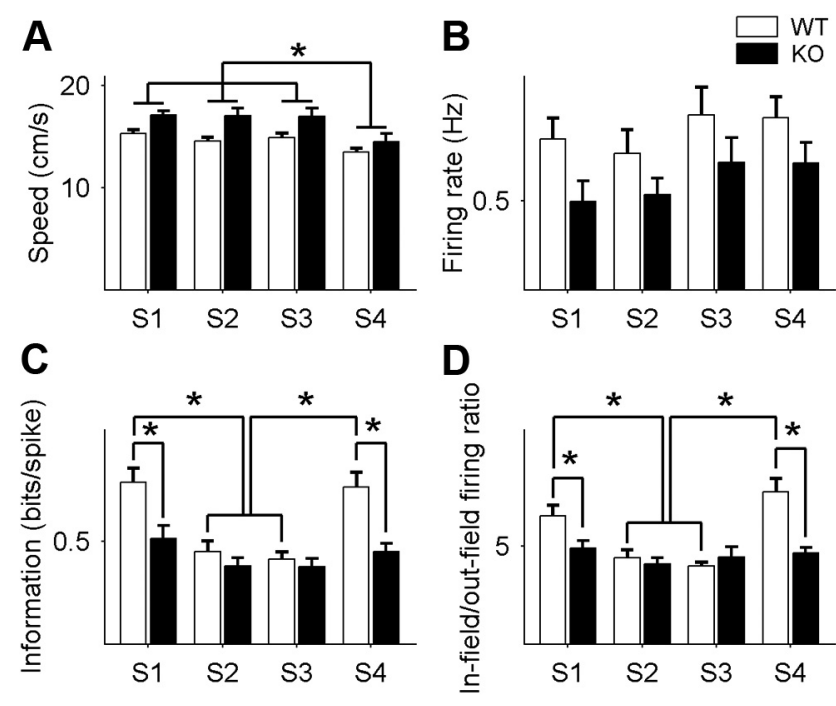

Figure 2. Selectivity of spatial firing. $\boldsymbol{A}$, Mean running speed of WT and Bax-KO mice across sessions 1-4. $\boldsymbol{B}$, Mean firing rates of units recorded from WT and Bax-KO mice. $\boldsymbol{C}$, Spatial information content per spike. $\boldsymbol{D}$, In-field/out-field firing ratio. Error bars denote SEM. *Significant difference, $p<0.05$.

spatial information content per spike (Skaggs et al., 1993) as well as in-field/out-field firing ratio (the ratio between mean firing rate within a place field and that outside of it). To assess the stability of spatial firing during sessions 2 and 4 (30 min each), pixel-by-pixel correlations between the firing rate map of the first $10 \mathrm{~min}$ period and that of the final 10 min period were calculated and then transformed to Fisher's $z$ for normalization as previously described (Song et al., 2005). Only those session sets in which the animals visited $>95 \%$ of all arena pixels during all four sessions were included in the analysis (mean occupancy rate, light condition, WT, $99.7 \pm 0.1$; Bax-KO, $99.6 \pm 0.1$; dark condition, WT, $99.8 \pm$ 0.0, Bax-KO, $99.7 \pm 0.1 \%)$.

Statistical tests. Two-way ANOVA (within- or between-subjects where appropriate) and Bonferroni post hoc tests were used for statistical comparisons. A $p$ value $<0.05$ was used as the criterion for a significant statistical difference. All data are expressed as mean \pm SEM.

\section{Results}

\section{Selectivity of spatial firing}

Total 44 and 40 complex spike cells recorded from WT and Bax-KO mice, respectively, with mean firing rate $>0.1 \mathrm{~Hz}$ in at least one of four recording sessions, were subjected to analysis. Basic characteristics of CA1 spatial firing in WT and Bax-KO mice have been reported previously (Lee et al., 2009). In the present study, we focused on the selectivity and stability of CA1 spatial firing. Mean running speed was slightly higher in Bax-KO mice, as previously reported (Lee et al., 2009), and it decreased somewhat in session 4 (main effect of genotype, $F_{(1,32)}=6.405, p=0.016$; main effect of session, $F_{(3,96)}=25.573 ; p<0.001$; genotype $\times$ session interaction, $F_{(3,96)}=2.439, p=0.069$; significant difference between sessions 1 and $4, p<0.001,2$ and 4, $p<0.001$, and 3 and 4, $p<0.001$; Fig. $2 A)$. There was no significant difference in mean firing rate between the two genotypes, although it significantly varied across sessions (main effect of genotype, $F_{(1,82)}=3.49, p=$ 0.066 ; main effect of session, $F_{(3,246)}=3.09 ; p=0.028$; effect of genotype $\times$ session interaction, $F_{(3,246)}=1.87, p=0.905$; no significant difference in post hoc pairwise comparisons; Fig. $2 B)$. The differences in mean running speed and mean firing rate did not affect the subsequent analysis results. Similar results were obtained after matching these variables across the genotypes and sessions (data not shown). 

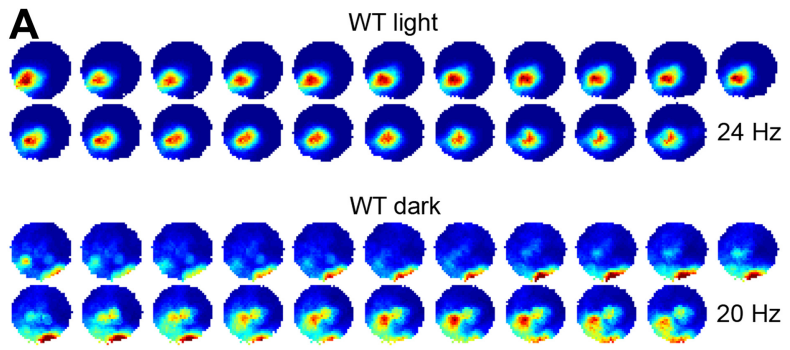

B
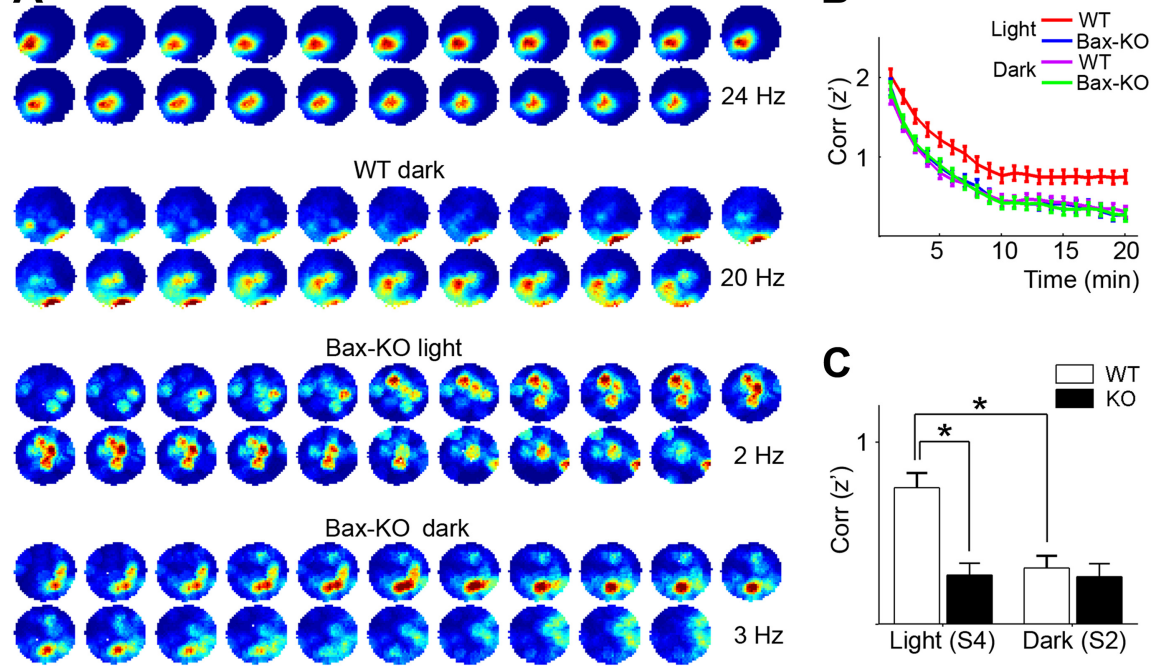

Figure 3. Stability of spatial firing. A, Examples of spatial firing over $30 \mathrm{~min}$ of recording in the light or dark (sessions 2 and 4 , respectively) for units recorded from WT or Bax-KO mice. Each spatial firing rate map was constructed based on neural activity during a 10 min time window that was advanced in 1 min steps (from left to right, top to bottom). The first rate map is for $0-10$ $\mathrm{min}$, the second one for 1-11 min, and so on. Red indicates the maximum firing rate that is different for each set of genotype/ illumination condition (indicated by numbers) and dark blue indicates no firing. $\boldsymbol{B}$, Time course of correlation ( $\boldsymbol{z}$-transformed) between the spatial firing rate map during the first $10 \mathrm{~min}$ period and that during a $10 \mathrm{~min}$ time window advanced in $1 \mathrm{~min}$ steps for each set of genotype/illumination condition. C, Mean correlation between the spatial firing rate map during the first 10 min and that during the last $10 \mathrm{~min}$ time period for each set of genotype/illumination condition. Error bars denote SEM. *Significant difference, $p<0.05$.

Spatial information content per spike was significantly higher in the WT animals recorded in the light than the other sets of genotype/illumination condition (WT/dark, Bax-KO/light, and Bax-KO/dark; main effect of genotype, $F_{(1,82)}=9.855, p=0.002$; main effect of session, $F_{(3,246)}=18.472, p<0.001$; effect of genotype $\times$ session interaction, $F_{(3,246)}=5.362, p=0.001$; significant difference between genotypes in sessions $1, p=0.005$, and 4 , $p<0.001$; significant difference between sessions 1 and $2, p<$ $0.001,1$ and $3, p<0.001,2$ and $4, p<0.001$, and 3 and $4, p<$ 0.001 in WT animals; Fig. $2 C$ ). Similarly, when we confined our analysis to those units with place fields (WT, $n=32,31$, 33, and 30 ; $B a x-K O, n=32,26,32$, and 29 in sessions $1-4$, respectively), in-field/out-field firing ratio was significantly higher in the WT animals recorded in the light than the other sets of genotype/ illumination condition (main effect of genotype, $F_{(1,232)}=$ 12.520, $p<0.001$; main effect of session, $F_{(3,232)}=9.599, p<$ 0.001 ; effect of genotype $\times$ session interaction, $F_{(3,232)}=5.618$, $p=0.001$; significant difference between genotypes in sessions 1 , $p=0.009$, and $4, p<0.001$; significant difference between sessions 1 and $2, p=0.005,1$ and $3, p<0.001,2$ and $4, p<0.001$, and 3 and $4, p<0.001$ in WT animals; Fig. $2 D)$.

\section{Stability of spatial firing}

We analyzed sessions 2 and 4 (30 min each) to examine stability of spatial firing over time. Only those neurons with mean firing rates $>0.1 \mathrm{~Hz}$ were included in the analysis (WT mice, 43 and 36 units in the light and dark condition, respectively; Bax-KO mice, 36 ad 29 units in the light and dark condition, respectively). The pattern of spatial firing changed over time in all recording sessions. However, the amount of change was significantly smaller for those units recorded from the WT animals in the light compared with the other sets of genotype/illumination condition (spatial firing rate map correlation between the first and last 10 min time periods, main effect of genotype, $F_{(1,140)}=12.654$, $p=0.001 ;$ main effect of session, $F_{(1,140)}=$ 9.214, $p=0.003$; effect of genotype $\times$ session interaction, $F_{(1,140)}=8.441, p=$ 0.004 ; significant difference between genotypes in session $4, p<0.001$; significant difference between illumination conditions in WT animals, $p<0.001$; Fig. 3 ).

\section{Discussion}

We examined effects of visual input on spatial firing of CA1 neurons using Bax-KO mice. Both selectivity and stability of spatial firing were dependent on the genotype as well as the illumination condition. They were significantly higher in the light than dark in WT animals. By contrast, in Bax-KO mice, they were similarly low in both illumination conditions. These results indicate that visual input allows spatial firing of CA1 neurons to be selective and stable, and that disruption of DG circuitry blocks these effects of visual input on spatial firing of CA1 neurons.

\section{Bax-KO mice as an animal model for specific disruption of DG circuit} We used Bax-KO mice as a model for selective disruption of DG circuit. Bax is a protein mediating apoptotic cell death in the nervous system (Oltvai et al., 1993; Deckwerth et al., 1996). Because Bax function is essential for the execution of cell elimination following adult neurogenesis in the DG, Bax-KO mice exhibit age-dependent accumulation of extra neurons in the DG (Sun et al., 2004). These extra DG neurons appear to intercalate with normal DG connections, and adult Bax-KO mice exhibit a selective disruption of synaptic transmission and synaptic plasticity in the DG (Kim et al., 2009; Lee et al., 2009). By contrast, other hippocampal circuits that do not include addition/elimination of new neurons in the adult are virtually normal. Brain functions independent on the hippocampal circuits, such as sensory and motor functions, are also intact (Buss et al., 2006; Kim et al., 2009; Lee et al., 2009), suggesting that the DG circuit is selectively impaired in adult Bax-KO mice. Therefore, $B a x-K O$ mice provide an opportunity to investigate effects of circuit disruption specific to the DG.

\section{Effect of visual input on the stability of spatial firing}

Spatial navigation based on internal cues (dead reckoning) is prone to cumulative error that can be corrected in reference to external landmarks (landmark-based navigation). Animals tend to rely on stable external landmarks over dead reckoning for spatial navigation when there is a conflict between the two (Gallistel, 1990). At the neural level, consistent with these behavioral findings, distal visual cues exert strong control over spatial firing of hippocampal place cells; when distal visual cues are rotated, place fields tend to rotate in reference to the visual cues (O'Keefe and Conway, 1978; O'Keefe and Speakman, 1987; Muller and Kubie, 1987; Knierim et al., 1995). One can expect from these findings that hippocampal spatial firing will undergo gradual drift in the absence of external sensory cues. Our results obtained from WT animals are consistent with this prediction. Spatial firing of CA1 neurons underwent significantly larger drifts in the dark than the light condition. The selectivity of spatial firing was 
also lower in the dark. Stability and selectivity of spatial firing are closely related measures. Less stable spatial firing will lead to less selective spatial firing, although the converse is not necessarily true. It is possible that less selective spatial firing in WT mice in the dark is because of unstable spatial firing.

Previous physiological studies in rats have yielded inconsistent results on this matter. Quirk et al. (1990) reported similar patterns of hippocampal spatial firing across light and dark conditions during a random foraging task. Markus et al. (1994) reported less specific and less reliable hippocampal spatial firing in the dark on an eight-arm radial maze. Because light and dark conditions alternated across trials in this study, the observed differences in spatial firing were most likely due to the difference in the availability of visual cues. However, Save et al. (2000) found unstable hippocampal spatial firing only after removal of both visual and olfactory cues during a random foraging task, demonstrating an importance of olfactory cues in maintaining stability of hippocampal spatial firing. Our experimental condition in the dark (session 2) is similar to the visual cue-deprivation/intact olfactory-cue condition in Save et al. (2000). In their study, within-session spatial firing stability under the intact visual-cue condition was similar to that under the visual cue-deprivation/ intact olfactory-cue condition. Thus, self-generated olfactory cues might be more effective as a spatial reference for hippocampal neuronal activity in rats than mice. There are a number of differences in the experimental procedures that may have contributed to different outcomes in our and previous studies, however. Additional studies are needed to clarify how hippocampal spatial firing is affected by diverse sources of external sensory information in different animal species.

\section{Role of DG in aligning internal spatial representation to external landmarks}

Several different hypotheses have been advanced regarding functional roles of the DG, such as pattern separation (for review, see Morris et al., 2012; Schmidt et al., 2012). According to a relatively recent hypothesis, the DG plays an important role in aligning internally generated spatial representation to external landmarks by binding together spatial and nonspatial information provided by the medial and lateral EC, respectively (Redish and Touretzky, 1997; Hafting et al., 2005; O’Keefe and Burgess, 2005; Knierim et al., 2006; Witter and Moser, 2006; Gorchetchnikov and Grossberg, 2007; Leutgeb and Leutgeb, 2007). In this regard, a pharmacological study has shown that specific blockade of plasticity at medial versus lateral perforant path synapses onto DG neurons has dissociable effects on processing spatial versus nonspatial aspects of environmental change (Hunsaker et al., 2007). We also have shown that $B a x-K O$ mice are impaired in finding a target location based on visual cues and spatial firing of their hippocampal neurons tends to be maintained with respect to the recording room rather than the recording chamber containing a visual cue (Lee et al., 2009). These results provide rare empirical evidence for the hypothesized role of the DG in aligning internally generated spatial representation to external landmarks. Nevertheless, given the paucity of empirical studies on this matter, additional studies testing key predictions of the hypothesis are needed to prove or disprove the hypothesis.

In the present study, we sought to test a critical prediction of the hypothesis. If the DG is indeed involved in aligning internally generated spatial maps to external landmarks, the effect of visual input on preventing time-dependent changes in hippocampal spatial firing observed in WT animals would be lost in Bax-KO mice. Our results confirmed this prediction. It is remarkable that the stability of spatial firing was similarly low in the light and dark in Bax-KO mice. Therefore, the effect of visual input on spatial firing stability was completely abolished in $\mathrm{Bax}-\mathrm{KO}$ mice. It is also remarkable that the stability of spatial firing in Bax-KO mice was similar to that in WT animals under darkness. These are the results that exactly match the predictions of the hypothesis. Our results therefore provide another line of empirical evidence for the hypothesis. Considering the convergence of medial and lateral EC projections in the DG, which have been proposed to carry spatial and nonspatial information, respectively (Redish, 1999; Knierim et al., 2006; Eichenbaum et al., 2007; Kerr et al., 2007), the DG might be the main stage where diverse elements of episodic (Tulving, 2002) or episodic-like (Clayton and Dickinson, 1998; Eacott and Norman, 2004; Babb and Crystal, 2006) memory (what, where, and when) are integrated. It remains to be determined whether the DG is also involved in integration processes other than associating internal spatial maps with the information on external landmarks.

\section{References}

Babb SJ, Crystal JD (2006) Episodic-like memory in the rat. Curr Biol 16:1317-1321.

Buss RR, Sun W, Oppenheim RW (2006) Adaptive roles of programmed cell death during nervous system development. Annu Rev Neurosci 29:1-35.

Clayton NS, Dickinson A (1998) Episodic-like memory during cache recovery by scrub jays. Nature 395:272-274.

Deckwerth TL, Elliott JL, Knudson CM, Johnson EM Jr, Snider WD, Korsmeyer SJ (1996) BAX is required for neuronal death after trophic factor deprivation and during development. Neuron 17:401-411.

Eacott MJ, Norman G (2004) Integrated memory for object, place, and context in rats: a possible model of episodic-like memory? J Neurosci 24:1948-1953.

Eichenbaum H, Yonelinas AP, Ranganath C (2007) The medial temporal lobe and recognition memory. Annu Rev Neurosci 30:123-152.

Fuhs MC, Touretzky DS (2006) A spin glass model of path integration in rat medial entorhinal cortex. J Neurosci 26:4266-4276.

Gallistel CR (1990) The organization of learning. Cambridge: MIT.

Gorchetchnikov A, Grossberg S (2007) Space, time and learning in the hippocampus: how fine spatial and temporal scales are expanded into population codes for behavioral control. Neural Netw 20:182-193.

Hafting T, Fyhn M, Molden S, Moser MB, Moser EI (2005) Microstructure of a spatial map in the entorhinal cortex. Nature 436:801-806.

Hargreaves EL, Rao G, Lee I, Knierim JJ (2005) Major dissociation between medial and lateral entorhinal input to dorsal hippocampus. Science 308:1792-1794.

Hunsaker MR, Mooy GG, Swift JS, Kesner RP (2007) Dissociations of the medial and lateral perforant path projections into dorsal DG, CA3, and CA1 for spatial and nonspatial (visual object) information processing. Behav Neurosci 121:742-750.

Jung MW, Wiener SI, McNaughton BL (1994) Comparison of spatial firing characteristics of units in dorsal and ventral hippocampus of the rat. J Neurosci 14:7347-7356.

Kerr KM, Agster KL, Furtak SC, Burwell RD (2007) Functional neuroanatomy of the parahippocampal region: the lateral and medial entorhinal areas. Hippocampus 17:697-708.

Kesner RP (2007) A behavioral analysis of dentate gyrus function. Prog Brain Res 163:567-576.

Kim WR, Park OH, Choi S, Choi SY, Park SK, Lee KJ, Rhyu IJ, Kim H, Lee YK, Kim HT, Oppenheim RW, Sun W (2009) The maintenance of specific aspects of neuronal function and behavior is dependent on programmed cell death of adult-generated neurons in the dentate gyrus. Eur J Neurosci 29:1408-1421.

Knierim JJ, Kudrimoti HS, McNaughton BL (1995) Place cells, head direction cells, and the learning of landmark stability. J Neurosci 15:1648-1659.

Knierim JJ, Lee I, Hargreaves EL (2006) Hippocampal place cells: parallel input streams, subregional processing, and implications for episodic memory. Hippocampus 16:755-764.

Knudson CM, Tung KS, Tourtellotte WG, Brown GA, Korsmeyer SJ (1995) 
Bax-deficient mice with lymphoid hyperplasia and male germ cell death. Science 270:96-99.

Lee JW, Kim WR, Sun W, Jung MW (2009) Role of dentate gyrus in aligning internal spatial map to external landmark. Learn Mem 16:530-536.

Leutgeb S, Leutgeb JK (2007) Pattern separation, pattern completion, and new neuronal codes within a continuous CA3 map. Learn Mem 14:745-757.

Leutgeb S, Leutgeb JK, Barnes CA, Moser EI, McNaughton BL, Moser MB (2005) Independent codes for spatial and episodic memory in hippocampal neuronal ensembles. Science 309:619-623.

Markus EJ, Barnes CA, McNaughton BL, Gladden VL, Skaggs WE (1994) Spatial information content and reliability of hippocampal CA1 neurons: effects of visual input. Hippocampus 4:410-421.

McNaughton BL, Battaglia FP, Jensen O, Moser EI, Moser MB (2006) Path integration and the neural basis of the 'cognitive map'. Nat Rev Neurosci 7:663-678.

Molter C, Yamaguchi Y (2008) Enthorhinal theta phase precession sculpts dentate gyrus place fields. Hippocampus 18:919-930.

Morris AM, Churchwell JC, Kesner RP, Gilbert PE (2012) Selective lesions of the dentate gyrus produce disruptions in place learning for adjacent spatial locations. Neurobiol Learn Mem 97:326-331.

Muller RU, Kubie JL (1987) The effects of changes in the environment on the spatial firing of hippocampal complex-spike cells. J Neurosci 7:1951-1968.

O'Keefe J, Burgess N (2005) Dual phase and rate coding in hippocampal place cells: theoretical significance and relationship to entorhinal grid cells. Hippocampus 15:853-866.

O'Keefe J, Conway DH (1978) Hippocampal place units in the freely moving rat: why they fire where they fire. Exp Brain Res 31:573-590.

O'Keefe J, Nadel L (1978) The hippocampus as a cognitive map. Oxford: Clarendon.

O'Keefe J, Speakman A (1987) Single unit activity in the rat hippocampus during a spatial memory task. Exp Brain Res 68:1-27.

Oltvai ZN, Milliman CL, Korsmeyer SJ (1993) Bcl-2 heterodimerizes in vivo with a conserved homolog, Bax, that accelerates programmed cell death. Cell 74:609-619.

Quirk GJ, Muller RU, Kubie JL (1990) The firing of hippocampal place cells in the dark depends on the rat's recent experience. J Neurosci 10:2008-2017.

Redish AD (1999) Beyond the cognitive map: from place cells to episodic memory. Cambridge, MA: MIT.

Redish AD, Touretzky DS (1997) Cognitive maps beyond the hippocampus. Hippocampus 7:15-35.

Sargolini F, Fyhn M, Hafting T, McNaughton BL, Witter MP, Moser MB, Moser EI (2006) Conjunctive representation of position, direction, and velocity in entorhinal cortex. Science 312:758-762.

Save E, Nerad L, Poucet B (2000) Contribution of multiple sensory information to place field stability in hippocampal place cells. Hippocampus 10:64-76.

Schmidt B, Marrone DF, Markus EJ (2012) Disambiguating the similar: the dentate gyrus and pattern separation. Behav Brain Res 226:56-65.

Skaggs WE, McNaughton BL, Gothard KM, Markus EJ (1993) An information-theoretic approach to deciphering the hippocampal code. In: Advances in neural information processing systems (Hanson SJ, Cowan JD, Giles CL, eds), pp 1030-1037. San Mateo, CA: Morgan Kaufmann.

Song EY, Kim YB, Kim YH, Jung MW (2005) Role of active movement in place-specific firing of hippocampal neurons. Hippocampus 15:8-17.

Sun W, Winseck A, Vinsant S, Park OH, Kim H, Oppenheim RW (2004) Programmed cell death of adult-generated hippocampal neurons is mediated by the proapoptotic gene Bax. J Neurosci 24:11205-11213.

Treves A, Tashiro A, Witter MP, Moser EI (2008) What is the mammalian dentate gyrus good for? Neuroscience 154:1155-1172.

Tulving E (2002) Episodic memory: from mind to brain. Annu Rev Psychol 53:1-25.

Witter MP, Moser EI (2006) Spatial representation and the architecture of the entorhinal cortex. Trends Neurosci 29:671-678. 